\title{
Acute afterload increase causes mechanical dispersion - potential mechanism for pressure induced arrhythmias?
}

Nina Jakuš ${ }^{1 *}$, George R. Sutherland', Maja Čikeš ${ }^{2}$, Peter Haemers ${ }^{1}$, Jens-Uwe Voigt', Frank Rademakers', Rik Willems ${ }^{1}$, Jan D'hooge ${ }^{1}$, Piet Claus ${ }^{1}$

${ }^{1}$ Catholic University of Leuven, Department of Cardiology, Leuven, Belgium

${ }^{2}$ University of Zagreb School of Medicine, University Hospital Centre Zagreb, Zagreb, Croatia

Objectives: We have recently shown that an acute afterload increase of the left ventricle induces premature ventricular beats (PVB). We explored mechanical dispersion, which has recently been linked to a greater incidence of arrhythmias in post infarction ICD patients, as a potential underlying mechanism.

Methods: 7 closed chest, closed pericardium pigs were instrumented with an aortic balloon in the mid-thoracic descending aorta. Parasternal long-axis B-mode images were acquired before and during the balloon inflation ( $\mathrm{N}=22)$, causing a $30 \%$ increase in systolic blood pressure and an acute LV dilatation over 5-10 beats. Before and during inflation, segmental septal deformation was analyzed using speckle tracking and timing and the amount of peak strain were measured. Mechanical dispersion was calculated as the standard deviation (SD) of the time to peak strain in the three segments. 12 lead ECG was acquired in all animals.

Results: PVBs occurred in $33 \%$ of the pressure challenges. The afterload increase caused a visible dispersion in the timing of the strain curves of the three segments (Figure 1a). Despite no change in QT interval, mean time of peak strain increased from $291 \pm 81 \mathrm{~ms}$, in preinflation to $352 \pm 56$ ms during inflation in the three segments, resulting in post- systolic shortening. The SD increased significantly from preinflation to inflation $(p=0.02)$ (Figure 1b). While strain at aortic valve closure clearly went down with afterload $(-14.74 \pm 6$ to $-11.04 \pm 4.02, p=0.0001)$, peak shortening remained simi$\operatorname{lar}(-15.79 \pm 6.09$ to $-15.12 \pm 4.56, p=0.479)$.

Conclusion: Acute afterload increase of the LV significantly increased the difference in time to peak strain between the three septal segments, resulting in increased mechanical dispersion, which may play a role in pressure-induced arrhythmias.

KEYWORDS: acute afterloading, premature ventricular beats, porcine model.

CITATION: Cardiol Croat. 2013;8(9):305-306.

Received: $30^{\text {th }}$ Jul 2013

*Address for correspondence: Catholic University of Leuven, Department of Cardiology, 3000 Leuven, Belgium.

Phone +385-91-560-5795

E-mail: nina.jakush@gmail.com

Figure 1a. Strain curves for the three segments in preinflation and inflation.

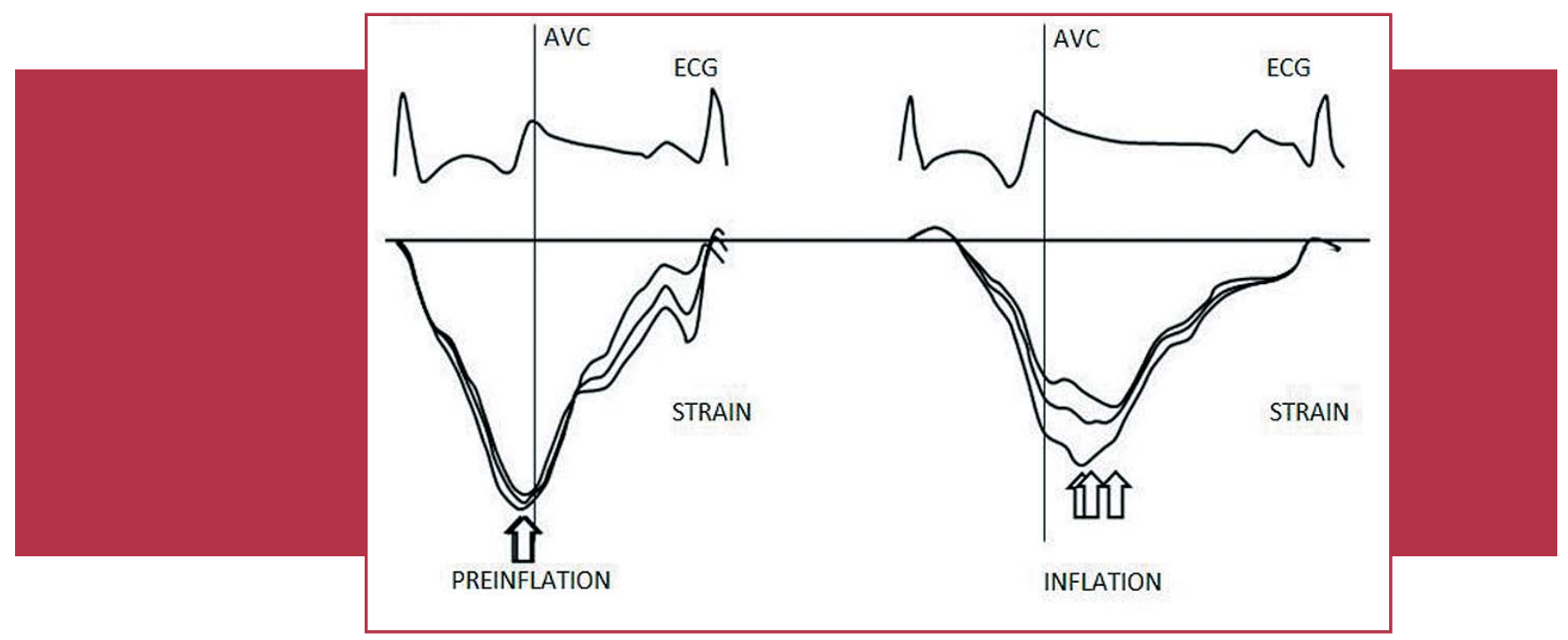


Figure $1 \mathrm{~b}$. Standard deviation of the time to peak strain between the three segments in preinflation and inflation.

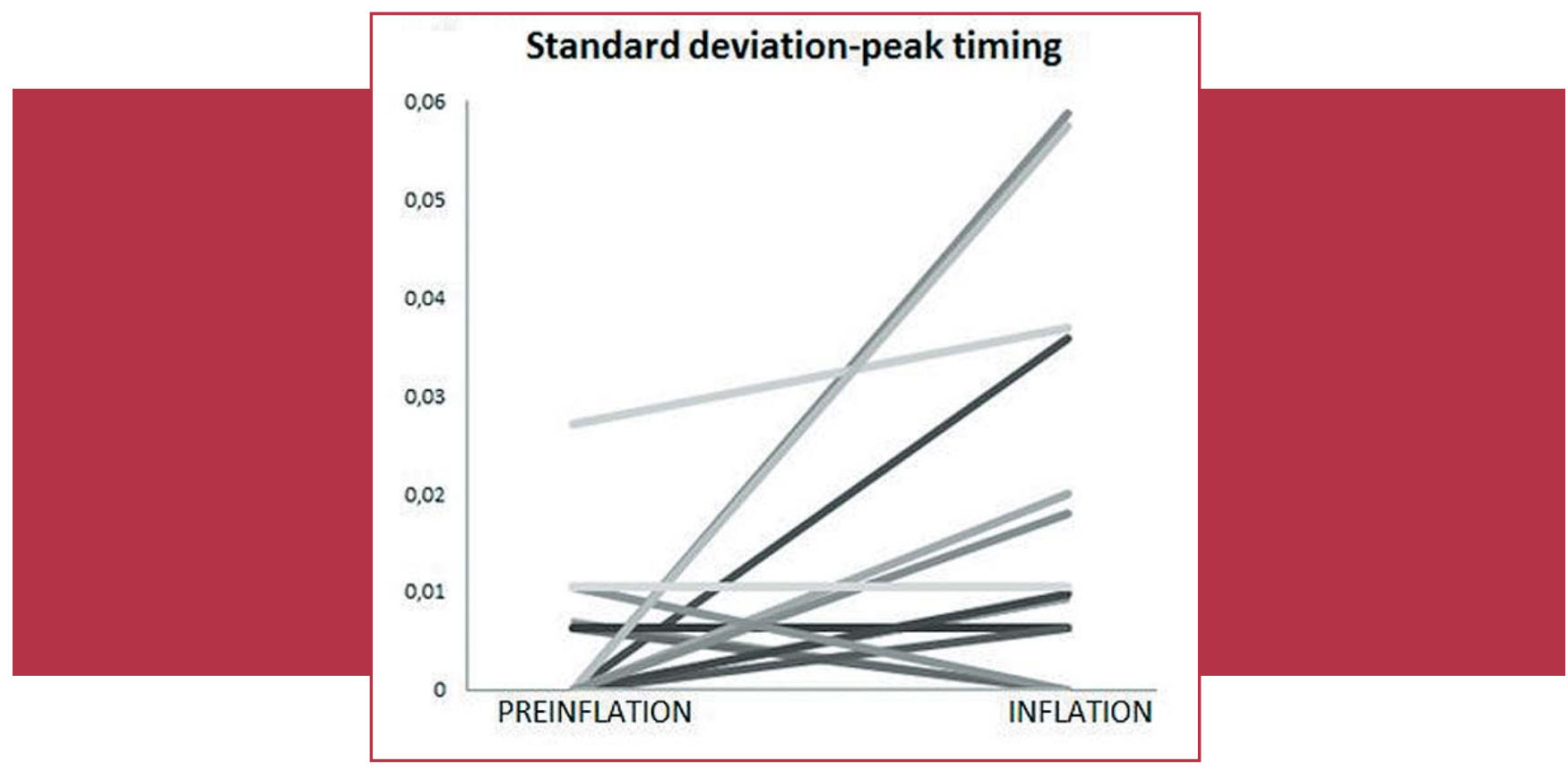

\section{Literature}

1. Gillebert TC, Lew WY. Influence of systolic pressure profile on rate of left ventricular pressure fall. Am J Physiol. 1991;261(3 Pt 2):H805-13.

2. Haugaa KH, Smedsrud MK, Steen T, et al., Mechanical dispersion assessed by myocardial strain in patients after myocardial infarction for risk prediction of ventricular arrhythmia. JACC Cardiovasc. Imaging. 2010;3(3):247-56.

3. Greve G, Lab MJ, Chen R, et al. RV distension alters monophasic action potential duration during pulmonary arterial occlusion in anaesthetized lambs: evidence for arrhythmogenic RV mechanoelectrical feedback. Exp Phys. 2001;86:5:651-7. 